\title{
Mathematical model of caprine arthritis encephalitis considering the seasonal breeding
}

\author{
Teppei Hirata $^{1}$, Yoshihito Yonahara ${ }^{1}$, Faramarz Asharif ${ }^{1}$, Takeshi Miyagi ${ }^{2}$, Tsutomu Omatsu ${ }^{2}$, \\ Yasushi Shiroma $^{3}$, Tetsuya Mizutani ${ }^{2}$, Yasunori Nagata ${ }^{3}$, Shiro Tamaki ${ }^{1}$ \\ ${ }^{1}$ Department of Information Engineering, Faculty of Engineering, University of the Ryukyus, Nishihara town, Japan \\ ${ }^{2}$ Research and Education Center for Prevention of Global Infectious Disease of Animals, Faculty of Agriculture, Tokyo University of \\ Agriculture and Technology, Tokyo, Japan \\ ${ }^{3}$ Department of Electrical and Electronic Engineering, Faculty of Engineering, University of the Ryukyus, Nishihara town, Japan
}

\section{Email address:}

teppei@neo.ie.u-ryukyu.ac.jp (T. Hirata), yosihito@neo.ie.u-ryukyu.ac.jp (Y. Yonahara), faramarz@neo.ie.u-ryukyu.ac.jp (F. Asharif), tmiyagi1130@gmail.com (T. Miyagi), tomatsu@cc.tuat.ac.jp (T. Omatsu), yasusi@neo.ie.u-ryukyu.ac.jp (Y. Shiroma), tmizutan@cc.tuat.ac.jp (T. Mizutani),ngt@eee.u-ryukyu.ac.jp (Y. Nagata), shiro@neo.ie.u-ryukyu.ac.jp (S. Tamaki)

\section{To cite this article:}

Hirata Teppei, Yoshihito Yonahara, Faramarz Asharif, Takeshi Miyagi, Tsutomu Omatsu, Yasushi Shiroma, Tetsuya Mizutani, Yasunori Nagata, Shiro Tamaki. Mathematical Model of Caprine Arthritis Encephalitis Considering the Seasonal Breeding.Animal and Veterinary Sciences.Vol. 2, No. 3, 2014, pp. 70-74. doi: 10.11648/j.avs.20140203.13

\begin{abstract}
This study was aimed at developing mathematical model of caprine arthritis encephalitis (CAE) and considering effective measures to prevent CAE's infection spread. This model took account of the fluctuation of infection rate due to seasonal breeding. New CAE model was constructed based on the sexually transmitted disease (STD) model. The new model was implemented the new function represented cyclical changes of infection rate to include the changes of the seasonal breeding. And definition equation of basic reproductive numbers $\left(\mathrm{R}_{0}\right)$ was constructed in reference previous study. In order to consider effective measures to prevent CAE's infection, the spread of CAE's infection was performed simulation.
\end{abstract}

Keywords: Caprine Arthritis Encephalitis (CAE), Mathematical Model, Simulation, Seasonal Breeding, Goat

\section{Introduction}

Recent years, the risk of domestic animal infectious disease is increasing with the increase in logistics by overseas. Recent climate change produces some risk, such as increase the occurrence of emerging infectious diseases, or such as expansion of the affected regions. The outbreak of foot-and-mouth disease in the United Kingdom caused a crisis in British agriculture in 2001. The crisis was estimated to have cost the United Kingdom 3.1 billion pounds[1].In addition, BSE, SARS, Avian Influenza and so on, the damage caused by domestic animal infectious disease has become increasingly serious.

From among the domestic animal infectious disease, we study for Caprine Arthritis Encephalitis. Caprine Arthritis Encephalitis (CAE) is an animal infectious disease of goats, which caused by a CAE virus (CAEV) that is lentivirus in the family Retroviridae. CAE occurs all over the world. The symptoms of CAE are varied. They are divided into three types, arthritis, encephalomyelitis and pneumonia.
For mature goat, abnormal gait and swelling of the carpal joint is observed in the initial symptoms and joint pain and swelling increases gradually, finally they will be difficulty walking and become inability stand. Young goats of 4 months or less can develop encephalomyelitis and pneumonia. Goats without symptoms can be hold the virus and be source of infection. There is no cure or vaccine in CAE. CAE infection can reduce the milk yield and effect on the quality of milk production. The CAEV transmission is mainly due to mother-to-child transmission through breast milk. And it has been reported that the case of infection by contact over a long period of time in same cage or pen [2]. Thus, CAEV has been found in semen [3], there is a possibility that there is an infection by mating. However this route has not been investigated further.

In order to prevent infection spread, it is necessary to predict accurately how to spread of CAE's infection. Therefore, we propose a mathematical model that represents the spread of CAE's infection. We created a mathematical model of CAE based on sexually transmitted 
disease (STD) model in human. This model is represented by differential equations and considering the fluctuation of infection rate due to seasonal breeding. We assume that CAE has infection by mating, and apply STD model. We introduce the function of vertical infection and the function of seasonally breading to CAE model, since STD model considers only the horizontal infection.

It is known that Alpine and Saanen species that have been bred as dairy mainly all over the world were sesonal breeding animals.Therefore, the mathematical model of $\mathrm{CAE}$ has been added the function to represent the seasonal breeding.

Our objective is to propose new mathematical model of CAE considering the seasonal breeding and to discuss the effective Epidemic prevention protocol.

\section{Mathematical Model}

In this section, we will introduce two mathematical models for infectious disease that are called SIR model and STD model, and describe new mathematical model represents the spread of CAE's infection. This model includes the function that represents the fluctuation of infection rate and the change of number of births per day.

\subsection{SIR Model}

It is known that spread of the infection can be modeled by a simple differential equation. There are various kinds of mathematical model of infectious disease. The simplest model is known as the SIR model.SIR model was proposed by W. O. Kermack and A. G. McKendrick in 1927 [4]. InSIR model, the population is divided into three categories, susceptible (S), infected (I), removed (R). The increase and decrease of the each category are represented by the differential equations as follows;

$$
\left\{\begin{array}{l}
\frac{d S}{d t}=-\beta S I \\
\frac{d I}{d t}=\beta S I-\gamma I \\
\frac{d R}{d t}=\gamma I
\end{array}\right.
$$

$\beta$ is the infectivity. $\gamma$ is the recovery rate or the isolation rate.

" $\beta S I "$ represents the number of non-infected becomes infected per unit time. " $\gamma I$ " represents the number of infected quarantined, or the number of infected dead by disease per unit time.

\subsection{STD Model}

Infection route of $\mathrm{CAE}$ is vertical infection through breast milk and a horizontal infection by sexual intercourse. This feature is very similar to the case of STD in humans.Therefore, by referring to the mathematical model of STD, CAE model might be proposed.

Hashigohas proposed a mathematical model for the infection of sexually transmitted disease (STD) [5].In this model, the population is divided into 4 categories, men of infected(MS) and non-infected(MI), women of infected(FS) and non-infected(FI). STD model takes into account only the horizontal infection about adult male and female.

$$
\left\{\begin{array}{l}
M^{\prime}{ }_{S}=\lambda_{M}-\mu_{M} M_{S}-\beta_{M} c_{M} \frac{F_{I}}{F_{S}+F_{I}} M_{S}+f_{M} M_{I} \\
M^{\prime}{ }_{I}=-\left(\mu_{M}+\delta\right) M_{I}+\beta_{M} c_{M} \frac{F_{I}}{F_{S}+F_{I}} M_{S}-f_{M} M_{I} \\
F^{\prime}{ }_{S}=\lambda_{F}-\mu_{F} F_{S}-\beta_{F} c_{F} \frac{M_{I}}{M_{S}+M_{I}} F_{S}+f_{F} F_{I} \\
F_{I}^{\prime}=-\left(\mu_{F}+\delta\right) F_{I}+\beta_{F} c_{F} \frac{M_{I}}{M_{S}+M_{I}} F_{S}-f_{F} F_{I}
\end{array}\right.
$$

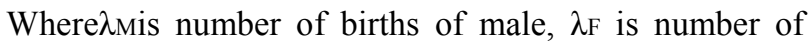
births of female, $\mu M$ is mortality rate of male, $\mu F$ is mortality rate of female, $\delta$ is direct mortality of infectious disease, $\beta \mathrm{M}$ is infection speed(female to male) and $\beta \mathrm{F}$ is infection speed(male to female), $\mathrm{cm}$ is frequency of sexual contact of men, $\mathrm{cF}$ is frequency of sexual contact of women, $f_{\mathrm{M}}$ is cure rate(male), $f_{\mathrm{F}}$ is cure rate(female).

\subsection{CAE Model}

We constructed the mathematical model of CAE based on SIR model and STD model. We used difference equations, because it is necessary to observe the change of goat per day in the rearing of goats. CAE also infect kid goat(prior to weaning) as well as mature goat unlike STD in human case, the population is divided into 6 categories including kid. We introduced growth rate and vertical infection rate to consider kid goat. CAE is incapable cured, it is not necessary to consider cure rate. CAE model is shown below;

$$
\left\{\begin{array}{l}
J_{S}^{\prime}=(1-e) b-\mu_{J} J_{S}-g J_{S} \\
J_{I}^{\prime}=e b-\left(\mu_{J}+\delta\right) J_{I}-g J_{I} \\
M^{\prime}{ }_{S}=\frac{1}{2} g J_{S}-\mu_{M} M_{S}-\beta_{M} c_{M} \frac{F_{I}}{F_{S}+F_{I}} M_{S} \\
M_{I}^{\prime}=\frac{1}{2} g J_{S}-\left(\mu_{M}+\delta\right) M_{I}+\beta_{M} c_{M} \frac{F_{I}}{F_{S}+F_{I}} M_{S} \\
F_{S}^{\prime}=\frac{1}{2} g J_{S}-\mu_{M} F_{S}-\beta_{F} c_{F} \frac{M_{I}}{M_{S}+M_{I}} F_{S} \\
F_{I}^{\prime}=\frac{1}{2} g J_{S}-\left(\mu_{F}+\delta\right) F_{I}+\beta_{F} c_{F} \frac{M_{I}}{M_{S}+M_{I}} F_{S}
\end{array}\right.
$$

Where JS is not-infected kid goat, JI is infected kid goat, MS is not-infected mature male goat, MI is infected mature male goat, FS is not-infected mature female goat, FI is infected mature female goat, $\mu \mathrm{J}$ is natural mortality of kid goat, $\mu \mathrm{M}$ is natural mortality of male goat, $\mu \mathrm{F}$ is natural mortality of female goat, $\delta$ is direct mortality by CAE, $\beta \mathrm{M}$ is infection speed(female to male goat) and $\beta \mathrm{F}$ is infection speed(male to female goat), $\mathrm{cM}$ is frequency of sexual 
contact of men, $\mathrm{cF}$ is frequency of sexual contact of women, $\mathrm{e}$ is vertical transition rate, $\mathrm{b}$ is total number of births of per time unit, $g$ is growth rate of per time unit.

In this study, we focused on seasonal breeding goats that mainly farmed in Japan. Therefore, the number of births per day is variable unlike human case. Goat has once or twice breeding season in a year. An increase of sexual contact in breeding season leads to an increase of horizontal infection, and vertical infection increases in production period. For this reason, considering breeding season in mathematical model is necessary to simulate CAE's infection spread with accuracy. Therefore the frequency of sexual contact(c) and the birthrate $(\eta)$ that fluctuate are defined as equations below.

$$
\begin{gathered}
b=\eta\left(F_{S}+F_{I}\right) \\
c=\left(\frac{1}{p}\right) \times\left(e^{a_{c}}-1\right) \\
a_{c}=\left(\left|\sin \left(2 \pi \times \frac{1}{T} \times(t-\theta)\right)\right|-\sin \left(2 \pi \times \frac{1}{T} \times(t-\theta)\right)\right)^{2} \\
\eta=\left(\frac{1}{p_{\eta}}\right) \times\left(e^{a_{\eta}}-1\right) \\
a_{\eta}=\left(\left|\sin \left(2 \pi \times \frac{1}{T} \times(t-\theta+150)\right)\right|-\sin \left(2 \pi \times \frac{1}{T} \times(t-\theta+150)\right)\right)^{2}
\end{gathered}
$$

The total number of $\operatorname{births}(b)$ is represented by using $\eta$. The $\operatorname{birthrate}(\eta)$ can be represented by plus 150 to phase $(\theta)$ of the function determining the $a_{c}$, because goat's pregnancy period is 150 days in average. In this model, there is once breeding season in a year. It is considered that the number of male's and female's sexual contact is equal, therefore we assumed $c_{\mathrm{M}}$ and $c_{\mathrm{F}}$ would be same.

Where $p$ is a parameter influencing to the peak value of the birth rate. $T$ is a parameter of breeding cycle. $\theta$ is a parameter influencing to phase.

Figure 1 is the graph represents the horizontal infection rate and the birthrate fluctuate cyclically. Here, we use the values as following to parameter; $p$ is $2000, T$ is $365, \theta$ is 7, $\beta M$ is $0.005, \beta F$ is 0.01 .

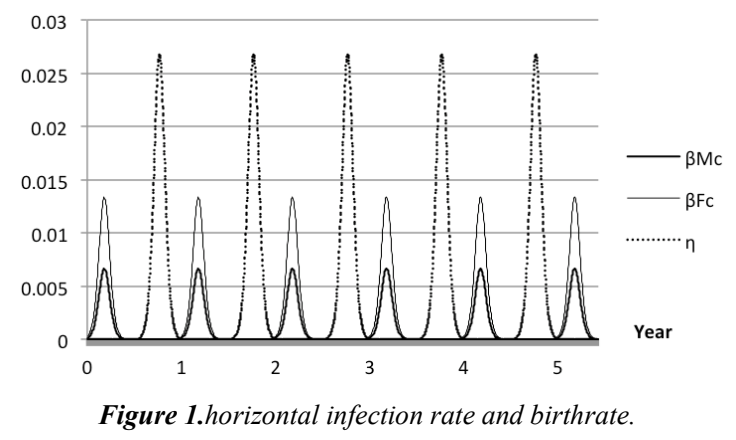

\section{Basic Reproduction Number}

\subsection{Introduction of $R_{0}$}

Basic reproduction number $\left(\mathrm{R}_{0}\right)$ is the expected value of the secondary infection which is reproduced by one infected person in entire infection period [4]. The infection spreads when $\mathrm{R}_{0}>1$, the infection comes to an end when $\mathrm{R}_{0}<1$. It is possible to compare the strength of infectivity from $\mathrm{R}_{0}$. In some cases, the convergence point of the infected exists even if $R_{0}>1 . R_{0}$ is represented as below equation.

$$
R_{0}=\frac{\beta S}{\gamma}
$$

$\beta$ is infection rate. $S$ is number of susceptible. $\gamma$ is recovery rate. " $\beta S$ " means number of secondary infected animalthat is infected by one infected animal per unit time. " $1 / \gamma$ " means the average time that infected animal is infection state.

Table 1 is an example of $\mathrm{R}_{0}$ about another infectious disease [6].

Table 1.Example of $R_{0}$ in some infectious disease of human and animals.

\begin{tabular}{ll}
\hline Disease & $\mathbf{R}_{\mathbf{0}}$ \\
\hline Influenza & $2 \sim 3$ \\
Mumps & $4 \sim 7$ \\
Smallpox & $5 \sim 7$ \\
Pertussis & $12 \sim 17$ \\
Measles & $12 \sim 18$ \\
Foot-and-mouth disease & 38 \\
\hline
\end{tabular}

This tabke refers to Fine (1993) [6]

\subsection{CAE's RO}

We constructed new CAE's definitional equation of $\mathrm{R}_{0}$ based on Macdonald model of AIDS. Macdonald model is non-density dependent type that is not proportional to the population. Goat does not look for the other sex even if there are many other sex, and if there are few other sex, goat looks for the other sex aggressively. For this reason, we used Macdonald model infection rate does not depend on the ratio of sex.CAE's definitional equation of $R_{0}$ is represented as equation below.

$$
R_{0}=\frac{\frac{\beta_{M} M_{S}+\beta_{F} F_{S}}{M_{S}+F_{S}} c+e \eta}{\frac{\mu_{J} J+\mu_{M} M+\mu_{F} F}{J+M+F}+\delta}
$$

Where $M_{S}$ is the number of susceptible male goats. $F_{S}$ is the number of susceptible female goats. $J, M, F$ are total number of each goat.

In $\mathrm{CAE}, \mathrm{R}_{0}$ is not constant value because infection rate is variable due to seasonal breeding. It is possible to find out when CAE's infection spreads by examining when $\mathrm{R}_{0}$ is larger than 1. 


\section{Simulation}

In order to discuss the effective quarantine protocol, we examined CAE's infection spread by simulation using CAE model. From result of simulation, we compare a case without CAE's infection to a case with CAE's infection, and find out when CAE's infection break out rapidly.

The difference equations used in CAE model was solved by the Euler's method, and spreadsheet software (Excel 2010, Microsoft) was used to obtain the numerical solution.

The values of the parameter used in simulation are shown in Table 2. A value of infection speed was set based on AIDS, because the epidemiological andmathematicalproperties of CAE were not reported. Growth rate of per time unit was set with reference that kid goat become mature goat in one year, and each natural mortality are estimated approximately values assuming that goat life is 15 years. Vertical infection rate and direct mortality rate by CAE are dummy because CAE's infection rate and direct mortality rate have not been investigated yet.

Initial values of each goat are shown in Table 3.

Table 2. Values of parameter.

\begin{tabular}{cll}
\hline Parameter & Mean & Value \\
\hline $\mathrm{e}$ & Vertical infection rate & 0.5 \\
$\mathrm{~g}$ & Growth rate of per time unit & 0.00274 \\
$\beta_{\mathrm{M}}$ & Infection speed (female to male) & 0.005 \\
$\beta_{\mathrm{F}}$ & Infection speed (male to female) & 0.01 \\
$\mu_{\mathrm{J}}$ & Mortality of kid goats & 0.00013 \\
$\mu_{\mathrm{M}}$ & Mortality of male goats & 0.00008 \\
$\mu_{\mathrm{F}}$ & Mortality of female goats & 0.00007 \\
$\delta$ & Direct mortality of infectious disease & 0.0005 \\
$\mathrm{p}$ & Peak value & 2000 \\
$\mathrm{~T}$ & Cycle & 365 \\
$\theta$ & Phase shift & 7 \\
\hline
\end{tabular}

Table 3. Values of parameter.

\begin{tabular}{cll}
\hline Parameter & Mean & Value \\
\hline $\mathrm{J}_{\mathrm{S}}$ & Susceptible kid goat & 200 \\
$\mathrm{~J}_{\mathrm{I}}$ & Infected kid goat & 20 \\
$\mathrm{M}_{\mathrm{S}}$ & Susceptible male goat & 400 \\
$\mathrm{M}_{\mathrm{I}}$ & Infected male goat & 40 \\
$\mathrm{~F}_{\mathrm{S}}$ & Susceptible female goat & 400 \\
$\mathrm{~F}_{\mathrm{I}}$ & Infected male goat & 40 \\
\hline
\end{tabular}

The graphs below show the result of simulation. Fig 1 is a case without CAE's infection. There is no infected in a case without CAE's infection, namely, infected kid goat $\left(J_{I}\right)$, infected male goat $\left(M_{I}\right)$ and infected female goat $\left(F_{I}\right)$ is 0 . Fig 2 is a case with CAE's infection.

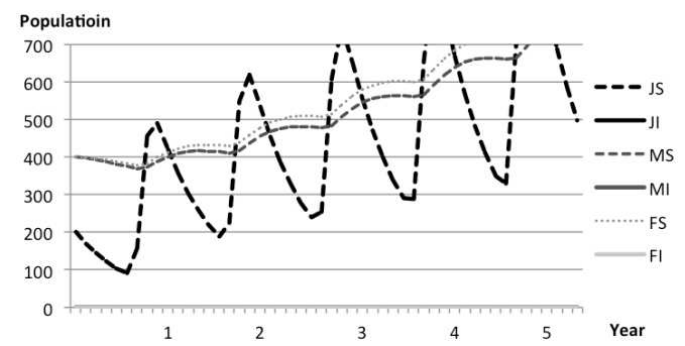

Figure 2. Without CAE's infection.

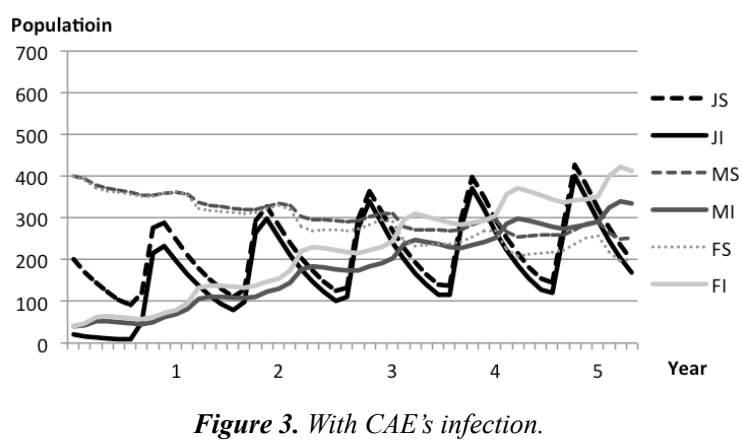

It can be confirmed that the decrease of the number of goat in comparison Fig 1 to Fig 2, since direct mortality increase by CAE's infection. Moreover, it can be confirmed that there is the period in which the spread of infection increase cyclically. This is due to seasonal breeding.

Fig. 3 is R0 led by the values of this simulation's parameter. The shaded area expresses the part of R0 is greater than 1, and these are mating season and delivery season.

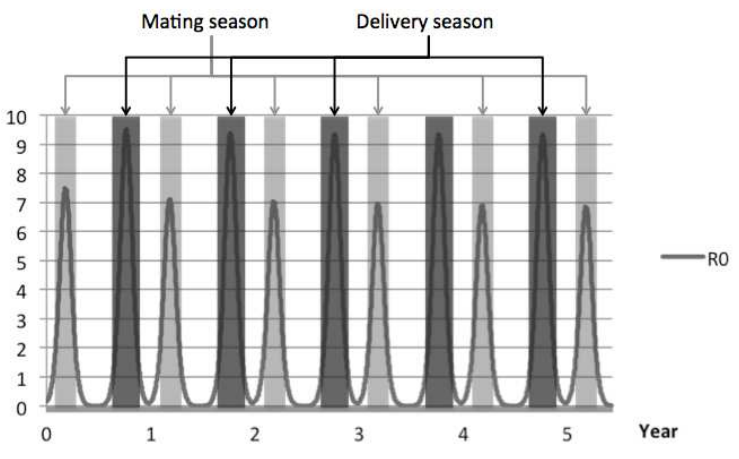

Figure 4. Fluctuation of $R_{0}$.

It was confirmed that the strength of infectivity was fluctuating cyclically from Fig 3 . In addition, the period in which is not only $\mathrm{R} 0$ is greater than 1 but also $\mathrm{R} 0<$ is smaller than 1 exist. In order to prevent infection, taking prevention measures when the period that $\mathrm{R} 0$ is greater than 1 is more effective. Because when R0 is smaller than 1 , spread of infection come to an end automatically.

Fig 4 shows the increase and decrease of infected and the period of R0 is greater than 1 . It can be confirmed that the infection increase when the part of $\mathrm{R} 0$ is greater than 1 .

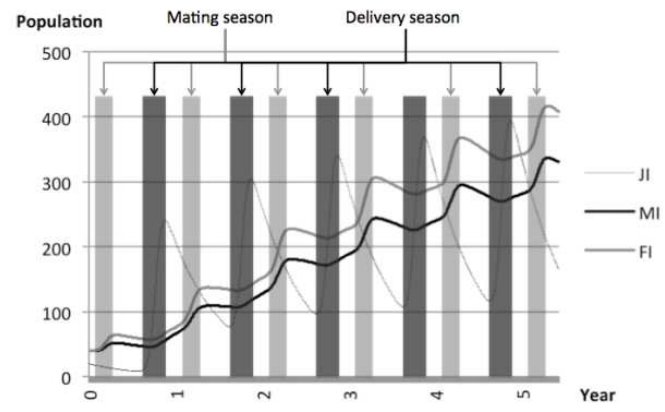

Figure 5. Fluctuation of $R_{0}$ and infected. 


\section{Conclusion}

In this study, we constructed the mathematical model of $\mathrm{CAE}$ and CAE's definitional equation of basic reproduction number (R0). It is necessary to consider that fluctuation of the infection rate in a case of goat unlike human case. Furthermore, using CAE model, we performed simulation to investigate how the CAE's infection spreads. From result of simulation, it was confirmed that the period that $\mathrm{R} 0$ is greater than 1 and R0 is smaller than 1 exist respectively. Infection come to an end when R0 is smaller than 1 . Therefore, taking prevention measures intensively when the period that $\mathrm{R} 0$ is greater than 1 is effective to prevent infection.

However, some values of parameter used in this simulation is dummy, therefore, It is necessary to investigate the values of parameter.

\section{Acknowledgements}

This study was conducted with the assistance of the Ministry of Internal Affairs and Communications of Japan by SCOPE scheme.Surge MIYAWAKI Co. Ltd., Okinawa Cross Head Co., Ltd.andNTTDATA CUSTOMER SERVICE Corporation. We would like to presence the appreciation.

\section{References}

[1] D. Thompson, P. Muriel, D. Russell, P. Osborne, A. Bromley,M. Rowland, S. Creigh-Tyteand C. Brown, "Economic costs of the foot and mouth diseaseoutbreak in the United Kingdom in 2001", Rev. sci. tech. Off. int. Epiz., 21 (3),2002, 675-687

[2] Adams, D.S., Klevjer, A.P., Carlson, J.L., McGuire, T.C., Gorham,J.R., "Transmission and control of caprine arthritisencephalitis virus". American Journal ofVeterinaryResrch,44,1983,pp.1670-1675

[3] K. Peterson, J. Brinkhof, D.J. Houwers, B. Colenbrander, B.M. Gadella "Presence of pro-lentiviral DNA in male sexual organs and ejaculates of small ruminant" Theriogenology 69, 2008, pp. 433-442

[4] Kermack W. O. and A. G. McKendrick, "A Contribution to the Mathematical Theory of Epidemics,"Proc. Roy. Soc. of London. Series A,1932, Vol. 115, No. 772

[5] Mathematical model of infectious disease, Baifukan (in Japanese), 2008,pp. 232-233.

[6] Paul E. M. Fine : "Herd Immunity", Epidemiologic Reviews, Vol.15, No.2 ,1993 